\title{
Relation of Accelerations in Two Inertial Frames in Special Relativity Theory
}

\author{
Sangwha-Yi* \\ Department of Math, Taejon University 300-716, South Korea
}

*Corresponding Author: Sangwha-Yi, Department of Math, Taejon University 300-716, South Korea

\begin{abstract}
In special relativity theory, we discover the relation of inertial frames'accelerations. In this theory, we can understand general state of the relation of inertial frames' accelerations
\end{abstract}

Keywords: Special Relativity theory, Acceleration, Inertial frame

PACS Number: 03.30. $+p$

\section{INTRODUCTION}

In special relativity theory, we discover the relation of inertial frames' accelerations.

At first, 4-dimension-Lorentz differential transformation is

$$
\begin{aligned}
& d \vec{x}=d \vec{x}+\gamma \vec{v}_{0} d t-(1-\gamma) \frac{\vec{V}_{0} \cdot d \vec{x}}{V_{0}^{2}} \vec{v}_{0} \\
& d t=\gamma\left(d t+\frac{\vec{V}_{0}}{c^{2}} \cdot d \vec{x}\right), \gamma=1 / \sqrt{1-\frac{V_{0}^{2}}{c^{2}}}
\end{aligned}
$$

If we rewrite $\mathrm{Eq}(1)-(2)$ in the other way,

$$
\begin{aligned}
& \frac{d \vec{x}}{d \tau}=\frac{d \vec{x}^{\prime}}{d \tau}+\gamma \vec{v}_{0} \frac{d t^{\prime}}{d \tau}-(1-\gamma) \frac{\vec{V}_{0}}{V_{0}{ }^{2}} \cdot \frac{d \vec{x}^{\prime}}{d \tau} \vec{V}_{0} \\
& d t=\gamma\left(d t^{\prime}+\frac{\vec{V}_{0}}{c^{2}} \cdot d \vec{x}\right)=\gamma d t^{\prime}\left(1+\frac{\vec{V}_{0}}{c^{2}} \cdot \vec{u}\right), \vec{V}=\frac{d \vec{x}}{d t}, \vec{u}=\frac{d \vec{x}^{\prime}}{d t^{\prime}}
\end{aligned}
$$

\section{The RELATION OF INERTIAL Frames'ACCELERATIONS}

For calculating the acceleration,

$$
\begin{aligned}
& \vec{a}=\frac{d}{d t}\left(\frac{d \vec{x}}{d \tau}\right)=\frac{1}{\gamma\left(1+\frac{\vec{v}_{0} \cdot \vec{u}}{c^{2}}\right)}\left[\frac{d}{d t^{\prime}}\left(\frac{d \vec{x}^{\prime}}{d \tau}\right)+\gamma \vec{v}_{0} \frac{d}{d t^{\prime}}\left(\frac{d t^{\prime}}{d \tau}\right)-(1-\gamma) \frac{\vec{v}_{0}}{v_{0}^{2}} \cdot \frac{d}{d t^{\prime}}\left(\frac{d \vec{x}^{\prime}}{d \tau}\right) \vec{V}_{0}\right] \\
& =\frac{1}{\gamma\left(1+\frac{\vec{v}_{0} \cdot \vec{u}}{c^{2}}\right)}\left[\vec{a}+\gamma \vec{v}_{0} \frac{d}{d t^{\prime}}\left(\frac{1}{\sqrt{1-\frac{u^{2}}{c^{2}}}}\right)-(1-\gamma) \frac{\vec{v}_{0} \cdot \vec{a}^{\prime}}{v_{0}^{2}} \vec{V}_{0}\right]
\end{aligned}
$$

In this time, the constant acceleration $a_{0}{ }^{\prime}$ is

$$
a_{0}^{\prime}=\frac{d}{d t^{\prime}}\left(\frac{u}{\sqrt{1-\frac{u^{2}}{c^{2}}}}\right) \rightarrow u=\frac{a_{0} t^{\prime}}{\sqrt{1+\frac{a_{0}{ }^{2} t^{2}}{c^{2}}}}
$$

Hence, 
$\left.\frac{d}{d t} \cdot\left(\frac{1}{\sqrt{1-\frac{u^{2}}{c^{2}}}}\right)=\frac{d}{d t^{\prime}}, \sqrt{1+\frac{a_{0}^{2} t^{2}}{c^{2}}}\right)=\frac{a_{0}}{c^{2}} \frac{a_{0} t^{\prime}}{\sqrt{1+\frac{a_{0}^{2} t^{2}}{c^{2}}}}=\frac{a_{0}^{\prime}}{c^{2}} u$

$\vec{v}_{0} \cdot \vec{u}=v_{0} u \cos \theta^{\prime}, \quad \vec{v}_{0} \cdot \vec{a}_{0}{ }^{\prime}=v_{0} a_{0}{ }^{\prime} \cos \theta^{\prime}$

Therefore, $\operatorname{Eq}(5)$ is

$$
\begin{aligned}
& \vec{a}=\frac{1}{\gamma\left(1+\frac{\vec{v}_{0} \cdot \vec{u}}{c^{2}}\right)}\left[\vec{a}_{0}+\gamma \vec{v}_{0} \frac{a_{0}{ }^{\prime}}{c^{2}} u-(1-\gamma) \frac{\vec{v}_{0} \cdot \vec{a}_{0}^{\prime}}{\left.v_{0}^{2} \vec{v}_{0}\right]}\right. \\
& =\frac{1}{\gamma\left(1+\frac{v_{0} u \cos \theta^{\prime}}{c^{2}}\right)}\left[\vec{a}_{0}+\gamma \vec{v}_{0} \frac{a_{0}^{\prime}}{c^{2}} u-(1-\gamma) \frac{v_{0} a_{0}{ }^{\prime} \cos \theta^{\prime}}{v_{0}^{2}} \vec{v}_{0}\right]
\end{aligned}
$$

If we calculate $\mathrm{Eq}(9)$,

$$
\begin{aligned}
& a^{2}=\vec{a} \cdot \vec{a}=\frac{1}{\gamma^{2}\left(1+\frac{v_{0} u \cos \theta^{\prime}}{c^{2}}\right)^{2}}\left[a_{0}{ }^{R}+\gamma^{2} v_{0}{ }^{2} \frac{a_{0}{ }^{R}}{c^{4}} u^{2}+(1-\gamma)^{2} a_{0}{ }^{R} \cos ^{2} \theta^{\prime}\right. \\
& \left.+2 a_{0}{ }^{R} \gamma v_{0} \cos \theta \cdot \frac{u}{c^{2}}-2 \gamma v_{0} \frac{a_{0}{ }^{R}}{c^{2}} u(1-\gamma) \cos \theta-2(1-\gamma) a_{0}{ }^{R} \cos ^{2} \theta^{\prime}\right] \\
& =\frac{1}{\left(1+\frac{V_{0} u}{c^{2}} \cos \theta^{\prime}\right)^{2}} a_{0}{ }^{R}\left[\frac{1}{\gamma^{2}}+\left(\frac{v_{0}^{2} u^{2}}{c^{4}}+2 \frac{V_{0} u}{c^{2}} \cos \theta+\frac{v_{0}^{2}}{c^{2}} \cos ^{2} \theta^{\prime}\right)\right]
\end{aligned}
$$

Finally, the relation of inertial frames' accelerations is

$$
a=\frac{\sqrt{\left(1-v_{0}^{2} / c^{2}\right)+\left(\frac{V_{0}^{2} u^{2}}{c^{4}}+2 \frac{V_{0} u}{c^{2}} \cos \theta+\frac{v_{0}^{2}}{c^{2}} \cos ^{2} \theta^{\prime}\right)}}{\left(1+\frac{V_{0} u}{c^{2}} \cos \theta^{\prime}\right)} a_{0}^{\prime}
$$

In special case, if $\theta^{\prime}=0$ in $\operatorname{Eq}(11),[1]$

$$
a=a_{0}{ }^{\prime}=\frac{d}{d t^{\prime}}\left(\frac{u}{\sqrt{1-\frac{u^{2}}{c^{2}}}}\right)=a_{0}=\frac{d}{d t}\left(\frac{V}{\sqrt{1-\frac{V^{2}}{c^{2}}}}\right), \vec{V}=\frac{d \vec{x}}{d t}, \vec{u}=\frac{d \vec{x}^{\prime}}{d t^{\prime}}
$$

$\mathrm{Eq}(11)$ is the general state of $\mathrm{Eq}(12)$.

According to the relativity principle, if we use ${ }^{-V_{0}, V}, \theta$ instead of $V_{0}, u, \theta^{\prime}$ in Eq(11), we can obtain the next relation of inertial frames' accelerations.

$$
a^{\prime}=\frac{\sqrt{\left(1-V_{0}^{2} / c^{2}\right)+\left(\frac{V_{0}{ }^{2} V^{2}}{c^{4}}-2 \frac{V_{0} V}{c^{2}} \cos \theta+\frac{V_{0}^{2}}{c^{2}} \cos ^{2} \theta\right)}}{\left(1-\frac{V_{0} V}{c^{2}} \cos \theta\right)} a_{0}
$$

In this time, the constant acceleration ${ }^{a_{0}}$ is 


$$
a_{0}=\frac{d}{d t}\left(\frac{V}{\sqrt{1-\frac{V^{2}}{c^{2}}}}\right), \vec{v}_{0} \cdot \vec{V}=v_{0} V \cos \theta, \quad \vec{v}_{0} \cdot \vec{a}_{0}=v_{0} a_{0} \cos \theta
$$

Additional special case, if $\theta=0$ in $\operatorname{Eq}(13),[1]$

$$
a=a_{0}=\frac{d}{d t}\left(\frac{V}{\sqrt{1-\frac{V^{2}}{c^{2}}}}\right)=a_{0}^{\prime}=\frac{d}{d t^{\prime}}\left(\frac{u}{\sqrt{1-\frac{u^{2}}{c^{2}}}}\right), \vec{V}=\frac{d \vec{x}}{d t}, \vec{u}=\frac{d \vec{x}^{\prime}}{d t^{\prime}}
$$

\section{CONCLUSION}

We can understand general state of the relation of inertial frames' accelerations in this theory.

\section{REFERENCES}

[1] S.Yi,"Expansion of Rindler Coordinate Theory and Light's Doppler Effect", The African Review of Physics, $8,37(2013)$

[2] S.Weinberg,Gravitation and Cosmology(John wiley \& Sons,Inc,1972)

[3] W.Rindler, Am.J.Phys.34.1174(1966)

[4] P.Bergman,Introduction to the Theory of Relativity(Dover Pub. Co.,Inc., New York,1976), Chapter V

[5] C.Misner, K,Thorne and J. Wheeler, Gravitation(W.H.Freedman \& Co.,1973)

[6] S.Hawking and G. Ellis, The Large Scale Structure of Space-Time(Cam-bridge University Press, 1973

[7] R.Adler,M.Bazin and M.Schiffer,Introduction to General Relativity(McGraw-Hill,Inc.,1965)

Citation: Sangwha-Yi, (2020). "Relation of Accelerations in Two Inertial Frames in Special Relativity Theory ". International Journal of Advanced Research in Physical Science (IJARPS) 7(2), pp.18-20, 2020.

Copyright: (C) 2020 Authors, This is an open-access article distributed under the terms of the Creative Commons Attribution License, which permits unrestricted use, distribution, and reproduction in any medium, provided the original author and source are credited. 